\title{
Investigation and Application of Zig-zag and Armchair Edged Graphene Nanoribbons in Nanoscale Electronic Devices
}

\author{
Nikolay Delibozov \\ Technical University of Sofia \\ 8 Kliment Ohridski Blvd, Sofia, Bulgaria \\ n.delibozov@ecad.tu-sofia.bg,nikolay_delibozov@yahoo.com
}

\begin{abstract}
This study investigates the roles of the edge and substrate effects of zig-zag and armchair graphene nanoribbons on atomic and electronic structures of graphene. Graphene is a two-dimensional flat monolayer of carbon atoms packed into a honeycomb lattice. It is made out of carbon atoms arranged in hexagonal structure. Graphene has a high electronic mobility and high charge carrier concentrations. Series of simulations with Zig-zag and Armchair nanoribbons are made using Gold, Copper, and Indium. The electronelectron interactions of the nanoribbons' molecules are calculated with generalized gradient approximation (GGA) through PerdewBurke-Ernzerhof (PBE) exchange correlation. The k-points are set to 1 x 1 x 50 for $\mathrm{n}_{\mathrm{a}}, \mathrm{n}_{\mathrm{b}}$, and $\mathrm{n}_{\mathrm{c}}$, respectively. The electron temperature of $300 \mathrm{~K}$ is used. The $\mathrm{C}-\mathrm{C}$ bond length between the carbon atoms in graphene nanoribbon is $1.24 \AA$. The superposition of the various orbitals in the nanoribbon leads to the variation in the Density of States (DOS). The Projected Density of States (PDOS) on the edge is mainly consistent around Fermi level. Electron Density calculations show regions with large electron density, which are found around the atoms, and their bonds. The observed nanotubes may have great potential for the application in nanoscale electronic devices fabrication and can be used as gate in the FET transistors.
\end{abstract}

Keywords: density of states, projected density of states, electron density, graphene nanoribbon, brillouin zone, fast-speed FET, nanoscale electronic device

\section{Introduction}

Graphene, the two-dimensional flat monolayer of carbon atoms packed into a honeycomb lattice, is of immense experimental as well as theoretical interest now due to its importance as building blocks for future carbon-based nanoscale electronic devices [1]. It is made out of carbon atoms arranged in hexagonal structure. Graphene has a high electronic mobility and high charge carrier concentrations. Its many unique properties are still not properly understood and need further investigations.

Since its discovery in 2004, graphene attracts focused attention and has growing interest of researchers due to its unique properties [2]. High carrier mobility in graphene at room temperature opens a wide range of its practical applications for production of nanoelectronic components and devices. This research work studies the roles of the edge and substrate effects of zig-zag and armchair graphene nanoribbons on atomic and electronic structures of interface build of zig-zag graphene nanoribbon (ZGNR) passivated with Gold and Copper, and armchair graphene nanoribbon (AGNR) passivated with Indium using the density functional theory (DFT) and generalized gradient approximation (GGA). Such nanoribbons can be used as a component of nanoscale electronic devices.

The ZGNR interface model is based on three-dimensional periodic slab scheme [2]. To simulate a system consisting of substrate and GNR, unit cells of 6 x 6 graphene is used. In order to build a zig-zag and armchair graphene nanoribbon (ZGNR and AGNR) Graphene cells are used. Fragments of the 6-ZGNR, 4-ZGNR and 4-AGNR slab are shown on Fig. 1. 

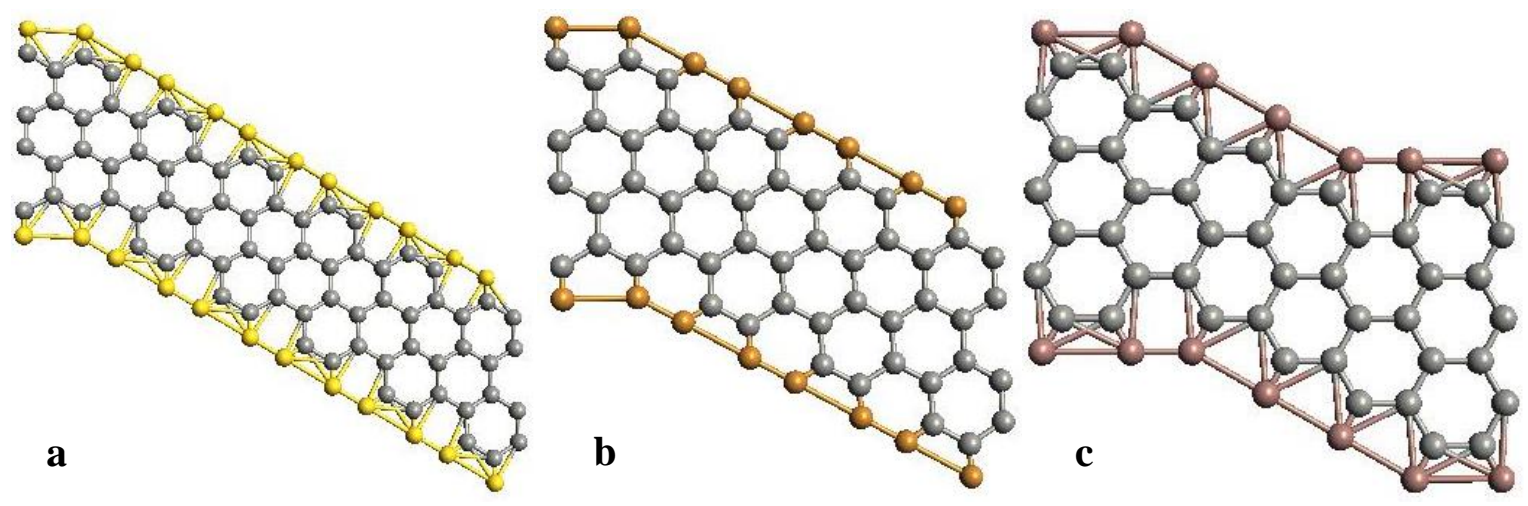

Fig. 1: Fragments of observed nanoribbons (a) Gold-terminated (b) Copper-terminated (c) Indium-terminated.

\section{Computational Methods}

Graphene nanoribbons have a peculiarity demonstrating non-linear dispersion relation for the low-energy spectrum of $\pi$-electrons [2]. Due to the nanoscale effect, nanoribbons are distinguished by $E_{\mathrm{g}}$ bandgap. Electron spectrum in zigzag-edged graphene nanoribbons (ZGNR) has specificity: existence of localized states determined by the boundary atoms on Fermi level.

DFT calculations are used for the computation of the observed nanoribbons. The electron-electron interaction of the molecules in the nanoribbons is calculated with generalized gradient approximation (GGA) through Perdew-BurkeErnzerhof (PBE) exchange correlation functional. The Brillouin zones are sampled with 1 x 1 x $50 \mathrm{k}$ points for $\mathrm{n}_{\mathrm{a}}, \mathrm{n}_{\mathrm{b}}$, and $\mathrm{n}_{\mathrm{c}}$, respectively.

The optimization of the measured area and the electron density of nanoribbon based device are performed by the double zeta polarized basis set for measured area and device electrode region [3]. The energy cutoff for real space mesh is set to $75 \mathrm{R}_{\mathrm{y}}$.

In the examined configuration, the $\mathrm{C}-\mathrm{C}$ bond length between the carbon atoms in graphene nanoribbon is amounted to $1.42 \AA$.

\section{Simulation Method}

As it is already mentioned all calculations are performed by the first-principles density functional theory (DFT). Norm-conserving pseudopotentials are used together with the double-zeta polarized basis set. Perdew-Burke-Ernzerhof generalized gradient approximations are applied to describe the exchange-correlation energy. Monkhorst-Pack k-point is set to $1 \times 1 \times 50$ in order to calculate the Brillouin zone integration. The electron temperature of $300 \mathrm{~K}$ is used.

The structure of zig-zag nanoribbons with the edge passivated by Gold and Copper are shown on Fig. $1 \mathrm{a}$ and b. The device consisting of armchair nanoribbons with edge passivated by Indium is shown on Fig. 1c. The structure of the heterostructures consists of the measured area and source and drain electrodes. These heterostructures can be used for application of logic transistor.

\section{Modeling and Results}

The systems under consideration are schematically shown in Fig. 2. 


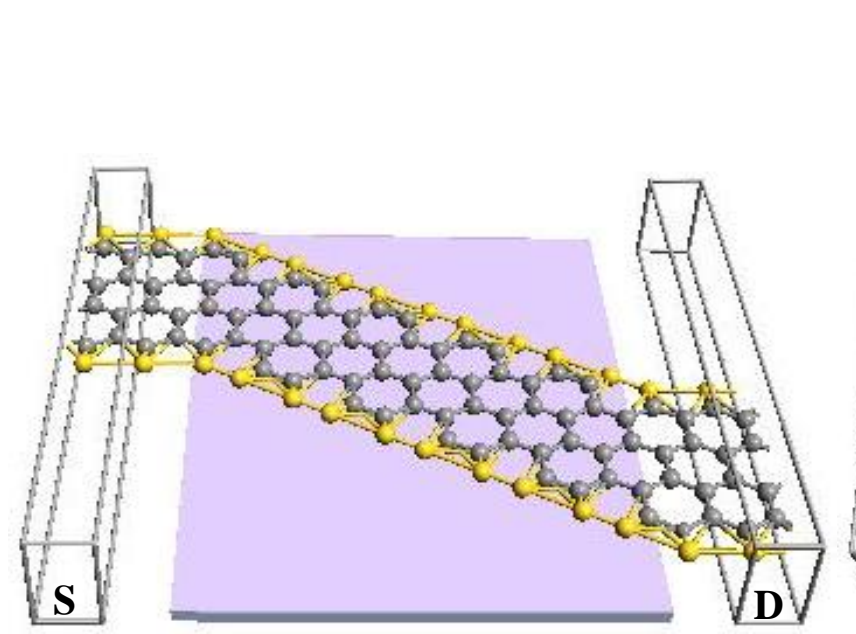

a

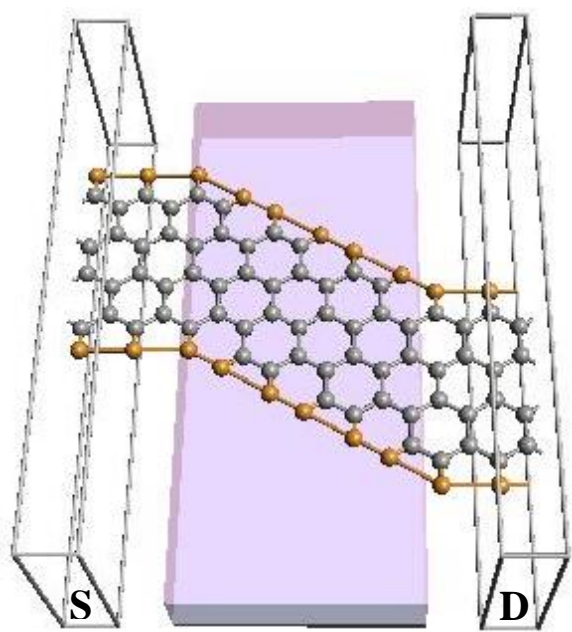

b

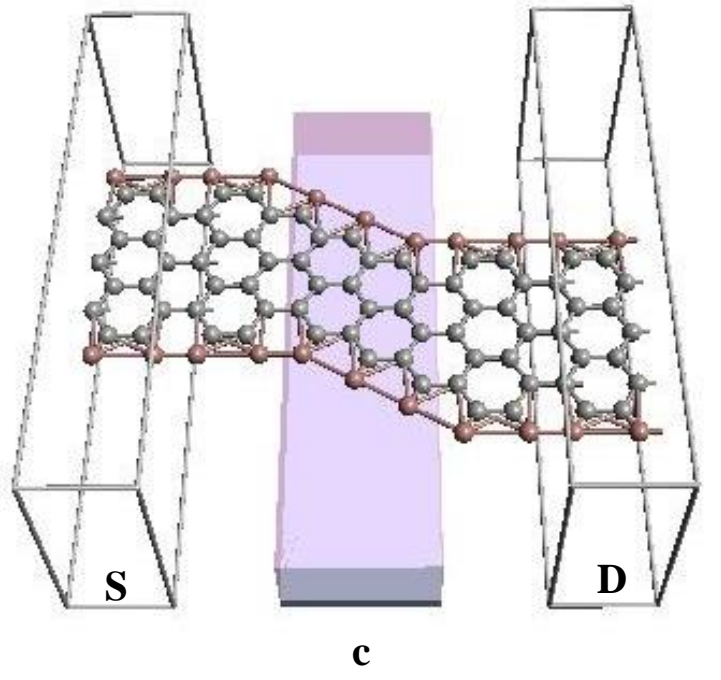

Fig.2: Device model structure based on nanoribbon (a) z-AuC nanoribbon (b) z-CuC nanoribbon (c) a-InC nanoribbon.

They consist of metallic electrodes linked to the GNR. The measured area of the nanoribbon is placed between the electrodes [4]. The first and second system corresponds to end- and side-contacted zig-zag GNRs, whereas the third one is armchair counterpart. The presented systems are fundamentally different from one another due to their atomic structure.

\subsection{Density of States}

The DOS spectrum provides the perception of the charge in the measured area. It will give information about density of the charge within the energy interval [5]. In this research work, the voltage across the two electrodes and the nanoribbon is varied within the interval of $0-1 \mathrm{~V}$. Here the Fermi level is zero. The device density of states (device DOS) will depends on the device projected density of states (device PDOS). The Gold atom has the electronic configuration of $1 s^{2} 2 s^{2} 2 p^{6} 3 s^{2} 3 p^{6} 3 d^{10} 4 s^{2} 4 p^{6} 4 d^{10} 5 s^{2} 5 p^{6} 4 f^{14} 5 d^{10} 6 s^{1}$, the Copper atom has the electronic configuration of $1 s^{2} 2 s^{2} 2 p^{6} 3 s^{2} 3 p^{6} 3 d^{10} 4 s^{1}$ and the Indium atom has the electronic configuration of $1 s^{2} 2 s^{2} 2 p^{6} 3 s^{2} 3 p^{6} 3 d^{10} 4 s^{2} 4 p^{6} 4 d^{10} 5 s^{2} 5 p^{1}$. The superposition of the various orbitals in the nanoribbon leads to the variation in the DOS (Fig. 3a, b, and c). 

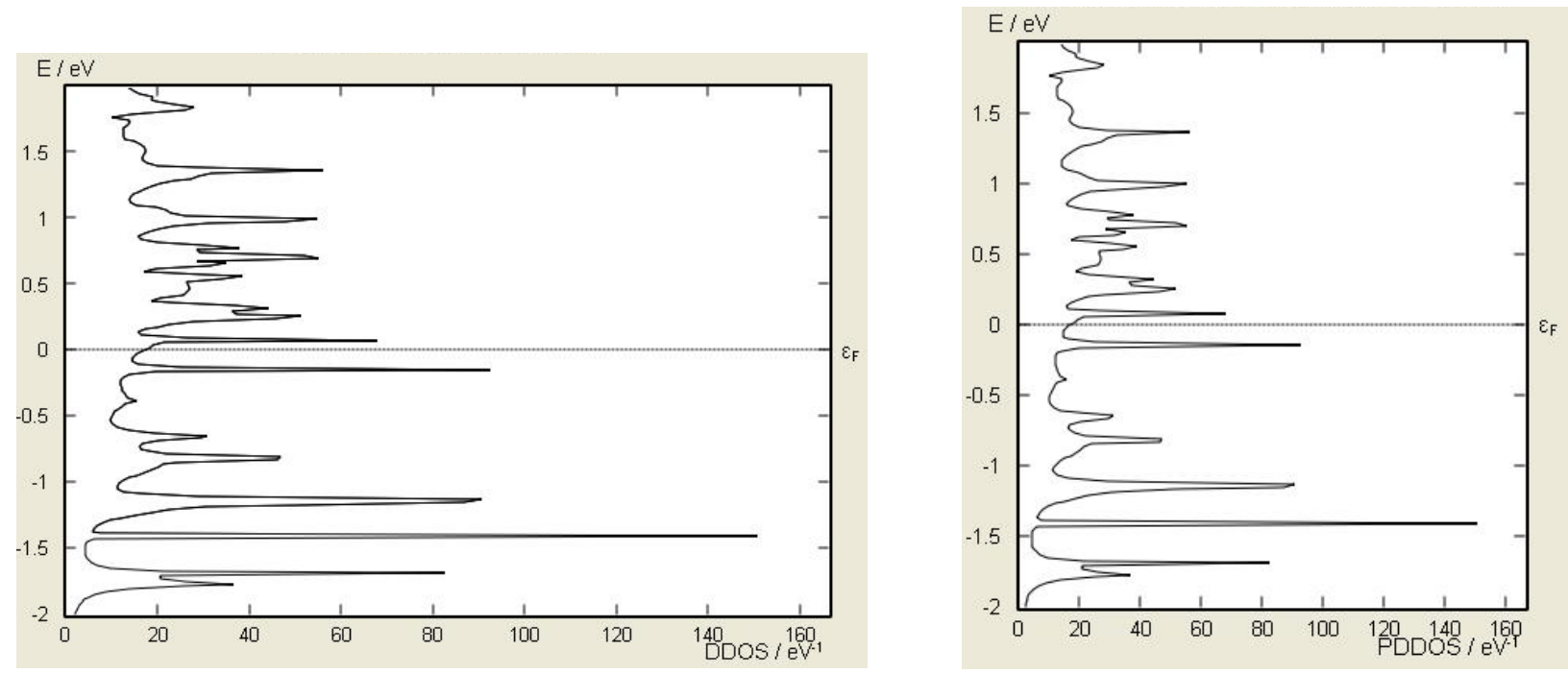

Fig. 3a: Density of States and Projected Density of States of nanoribbon device terminated with Gold.
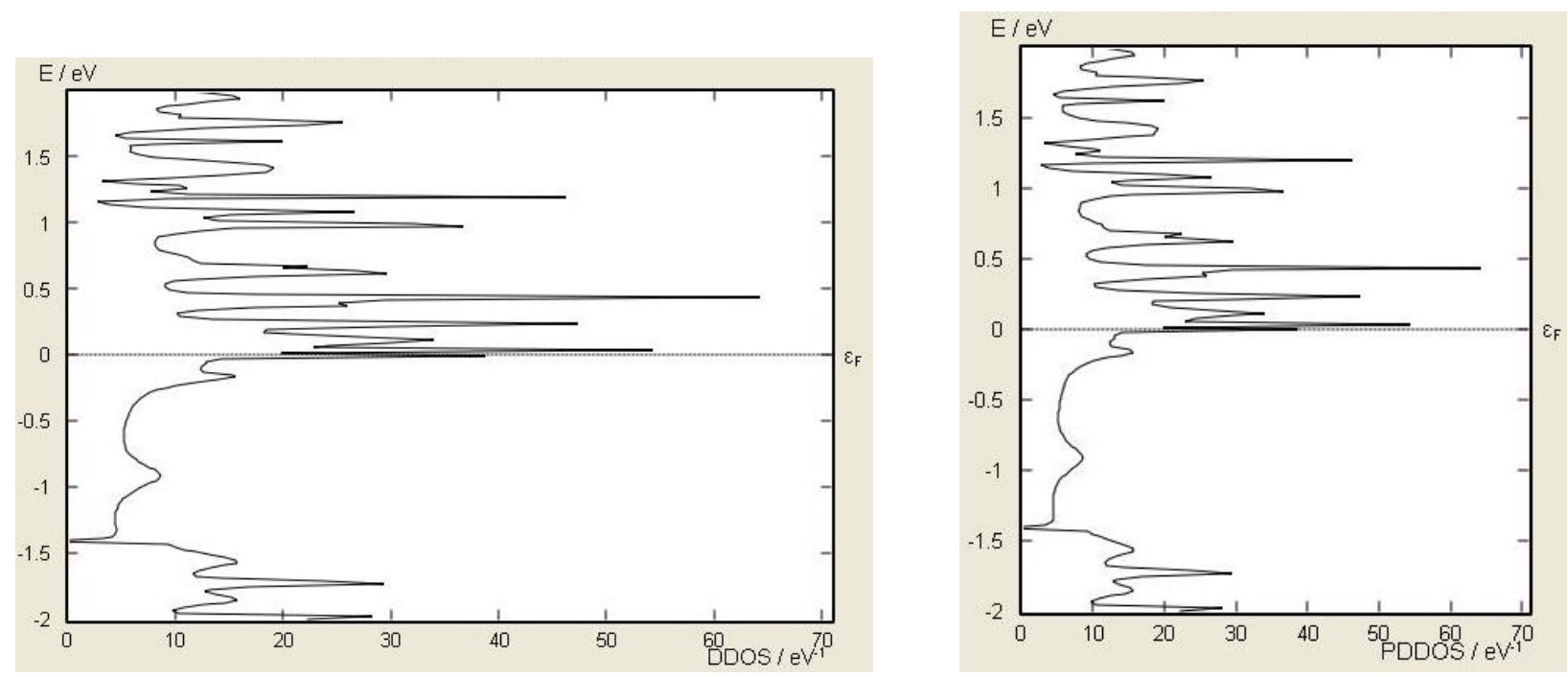

Fig. 3b: Density of States and Projected Density of States of nanoribbon device terminated with Copper. 

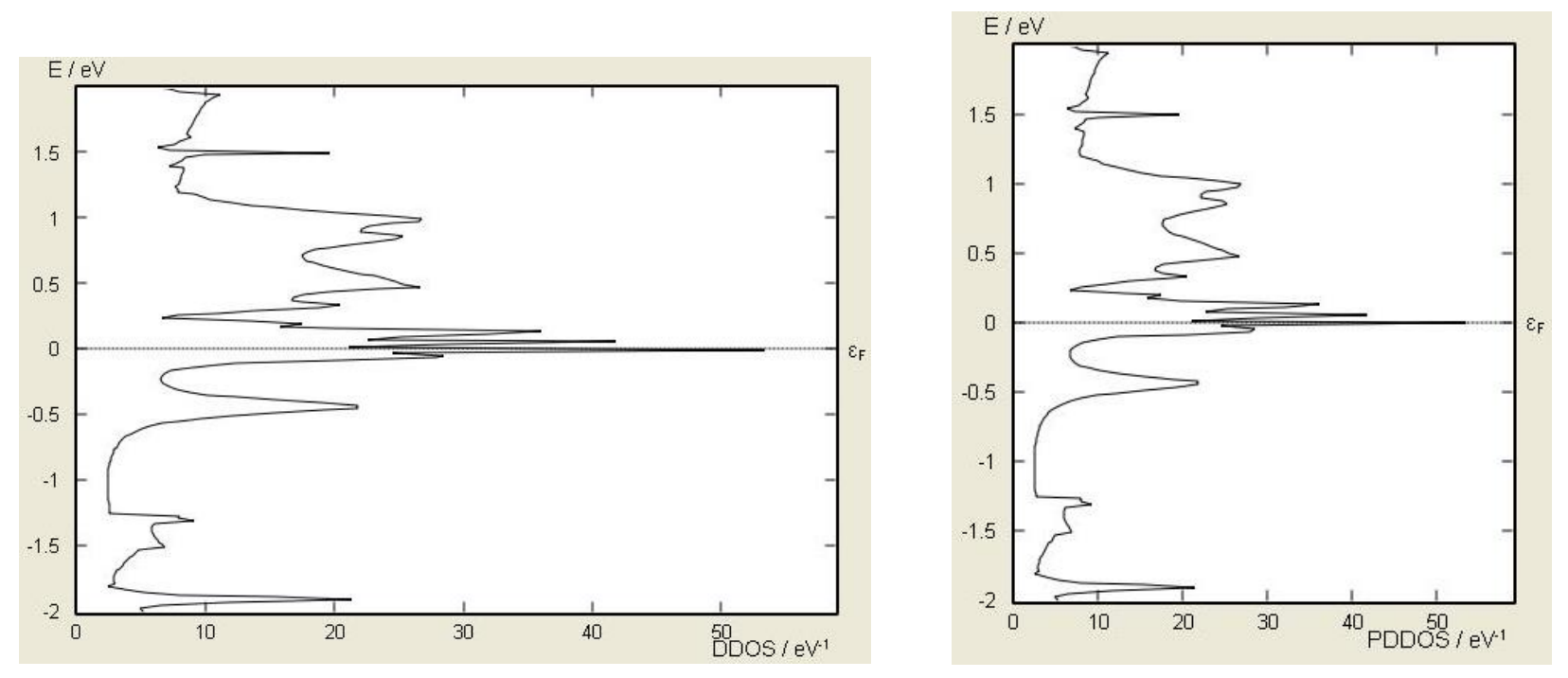

Fig. 3c: Density of States and Projected Density of States of nanoribbon device terminated with Indium.

The overlapping of the s orbitals leads to peak near $0.2 \mathrm{eV}$ in the positive energy above the Fermi level $\left(\mathrm{E}_{\mathrm{F}}\right)$ which refers to the conduction band in the device PDOS. The overlapping of the orbitals in the gold atom with the orbitals in the carbon leads to the more number of peaks in the positive energy of the spectrum. Inspecting the device DOS spectrum, more peaks can be seen in conduction band with a peak maximum at $1.4 \mathrm{eV}$.

When the bias voltage of $1 \mathrm{~V}$ is applied across the electrode, this perplexes the DOS across the nanoribbon, at zero bias condition. In device DOS spectrum localization of charge can be seen in the conduction band with more amplitude.

Interestingly, the projected density of states (PDOS) on the edge is mainly consistent around Fermi level, indicating that the states from the edges contribute to the electrons through the measured area.

Because the Indium atoms on the edges have higher energy with unfilled p orbit, the electron can easily be transported from them [2].

In another heterostructure the Indium atoms on the edge are replaced by Gold or Copper atoms. The Gold or Copper atoms on the edge have the higher energy, resulting in the electrons of Gold or Copper atoms hard transporting through the edge than from the inside.

\subsection{Electron Density}

Electron density is the measure of the probability of an electron being present at a specific location. According to quantum mechanics, due to the uncertainty principle on an atomic scale the exact location of an electron cannot be predicted, only the probability of its being at a given position.

In molecules, regions of large electron density are usually found around the atom, and its bonds.

Density functional theory (DFT) and the Hohenberg-Kohn theorems comprised a breakthrough for calculation methods in quantum chemistry. Basically the theorems say that the average number of electrons located at any one point in space.

As it is already mentioned the Electron Density is a representation of the probability of finding an electron in a specific location around an atom or molecule. In general, the electron is more likely to be found in regions with high electron density. Electron density of GNR with Gold, Copper and Indium atoms at the edge of the nanoribbon can be seen on Fig. 4a, b, and c. 

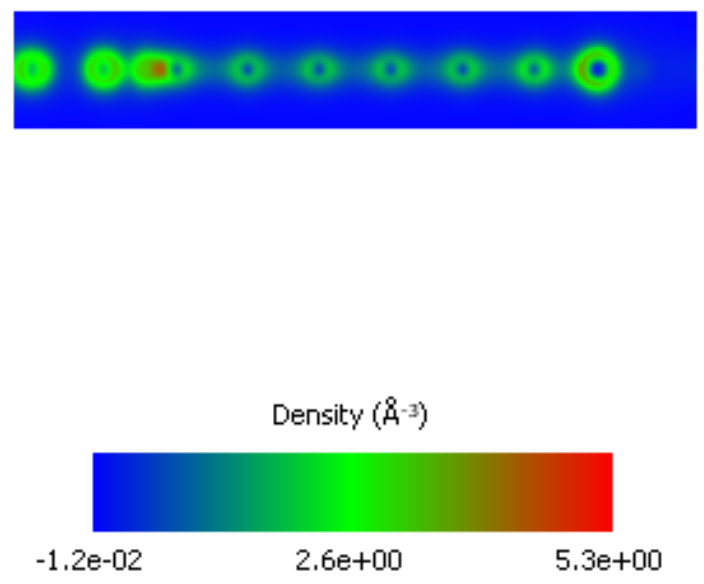

Fig. 4a: Electron density map of zig-zag nanoribbon with Gold atoms at the edge.
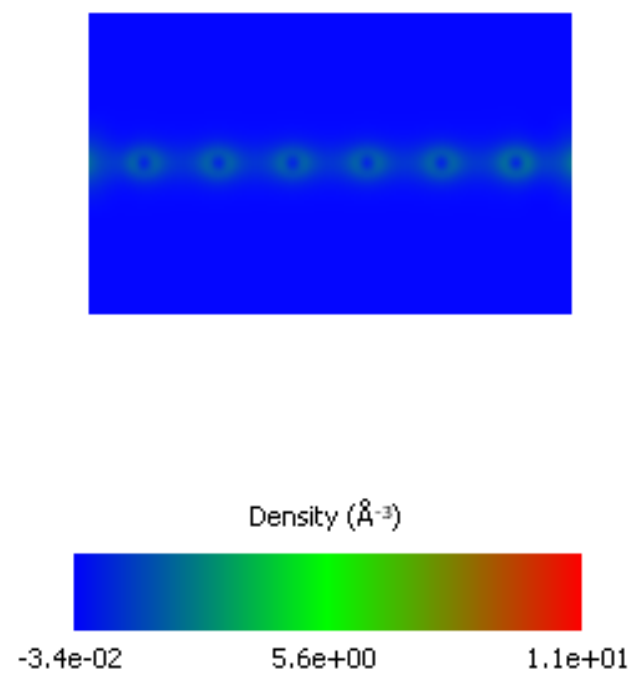

Fig. 4b: Electron density map of zig-zag nanoribbon with Copper atoms at the edge. 

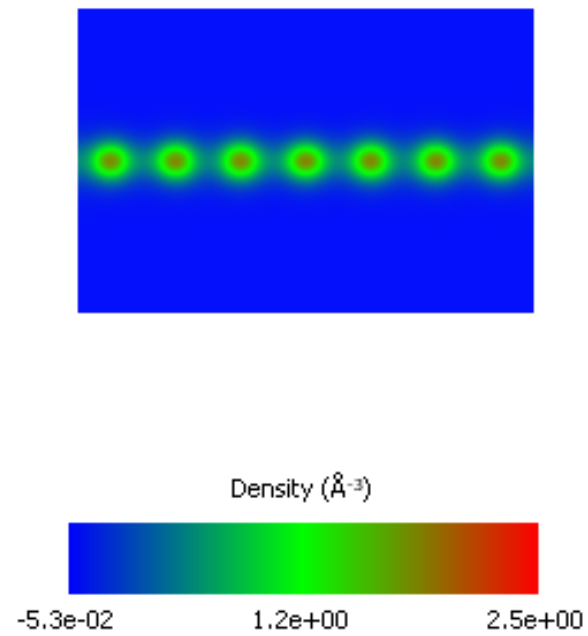

Fig. 4c: Electron density map of armchair nanoribbon with Indium atoms at the edge.

\section{Concluding Remarks}

First-principles density functional theory calculations have been conducted to explore the electronic properties of the graphene nanoribbons [2].

Due to the unique electronic properties, Electron Density and specific structure of graphene, such as the zero bandgap and its massless charge carriers, graphene-based nanoscale electronic devices have been attracting increasing attention. Many researchers focused on graphene field effect transistors (FETs) as a result that FETs are the fundamental unit of the integrated circuits [2]. Most researches have presented that graphene shows an extremely high carrier mobility, which enables it to easily fabricate into fast-speed FETs. It is well know that pristine graphene cannot be applied in the logic transistors as it does not possess a bandgap. Opening a sizable bandgap becomes an urgent issue for graphene. Cutting graphene into graphene nanoribbons (GNRs) is a solution that can help to solve the problem.

\section{Conclusion}

Graphene has acquired immense theoretical interest now. The size and geometry of the nanoscale carbon systems have important effects on their electronic properties [1]. In the present work, both the zig-zag and armchair grapheme nanoribbons (GNRs) were studied. Simple six sites lattice structure of graphene has been considered. Different edge conditions are imposed to model difference in edge geometries. Electron density at the edge-site and in-site has been calculated for both GNRs.

Interestingly with the various atoms-observed in this paper doping on the edges, graphene shows a fast current transport. Therefore, graphene may have the great potential for the application in logic transistor.

The results of topological analysis of the electron density in graphene nanoribbons with various edge passivated chemical elements (Gold, Copper, and Indium) based on the experimental - computer simulated (at 300K) were presented and discussed. This type of nanotubes can be used as gate (a central region) in the FET transistors. The features of the electron density lead to the conclusion that the $\mathrm{C}-\mathrm{Au}, \mathrm{C}-\mathrm{Cu}$ and $\mathrm{C}-\mathrm{In}$ interactions are of the partly polar covalent (or intermediate) type.

The two electrodes in the observed systems consist of two identical zig-zag and armchair graphene nanoribbons. The central part of each system is connected with the two electrodes. In this way a junction between electrodes and the central region is created.

The generalized gradient approximation (GGA) is used to evaluate exchange and correlation.

Appearance of an energy gap in the 6-ZGNR, 4-ZGNR and 4-AGNR interface opens certain opportunities for their use in components of graphene field-effect transistors and nanoscale electronic devices. 


\section{References}

[1] N. K. Ghosh, "Study of the Graphene Nanoribbons within Hubbard Hamiltonian.", Armenian Journal of Physics, vol. 6, issue 2, pp. 97-102, 2013.

[2] H. Li, Y. Zhou and J. Dong, "First-Principle Study of the Electron Transport Properties of Graphene-Like 2D Materials.", InTech, 2016, pp. 117-139.

[3] C. Motta, D. Sanchez-Portal, M. I. Trioni, "Transport properties of armchair graphene nanoribbon junction between graphene electrodes.", 2012.

[4] S. Krompiewski, "Graphene Nanoribbons with End- and Side-Contacted Electrodes.", Proceedings of the European Conference Physics of Magnetism, pp. 1216-1218, 2011.

[5] R. Chandiramouli, S. Sriram, "First-Principles Investigation on Electronic Transport Properties of Tungsten Nitride Nanoribbon Based Molecular Device.", Journal of Inorganic and Organometallic Polymers and Materials, pp. 737$744,2014$. 